surgical, particularly post-transphenoidal hypophysectomy, are common etiologies of OD and no effective treatments exist. The results from our pilot study will help better inform the best way to undergo OT, how effective it is, and the planning of future studies.

4569

\section{Burden of illness in idiopathic pulmonary fibrosis: A real-world cohort}

Erica Farrand ${ }^{1}$, and Harold Collard ${ }^{1}$

${ }^{1}$ University Of California, San Francisco

OBJECTIVES/GOALS: Studying IPF associated health care utilization (HRU) in real world settings, provides the opportunity to produce generalizable results that can directly inform models of care delivery. The objective of this study was to examine real-world differences in the natural history of annual HRU and treatment trends associated with IPF in a large, community-based population of patients with IPF, compared to matched non-IPF controls. METHODS/STUDY POPULATION: Cases of IPF were identified using case validated algorithms in the Kaiser Permanente Northern California adult population from 2000 to 2014. Each case was matched to at least one and no more than five non-IPF controls by age, sex, race/ethnicity and length of enrollment. The date of the first occurrence of the IPF-specific diagnostic code was considered the index date for cases and matched controls. Comorbidity burden and HRU was assessed in the five years pre- and post-index date, including hospitalizations, outpatient visits, use of diagnostic and monitoring studies and medications. Poisson generalized estimating equations models with robust standard errors were used to estimate adjusted case-control differences in HRU, accounting for clustering within matched sets. RESULTS/ANTICIPATED RESULTS: 691 patients were identified with incident IPF during the study period and matched to 3,452 control subjects. Demographics were well balanced between cases and controls due to matching. Patients with IPF had a higher burden of all selected co-morbidities and higher HRU compared to controls. In the pre-index period, IPF members had significantly higher rates of all diagnostic procedures $(p<0.001)$ and health care visits, with the exception of skilled nursing facility care $(\mathrm{p}<0.001)$. The greatest relative difference was observed with use of Chest CT $(R R=245.94,95 \%$ CI 117.04, 516.82). In the post-index period compared to controls, patients with IPF had higher rates of serial testing $(\mathrm{p}<0.001)$ and inpatient and outpatient care including, all-cause hospitalization (1.55), emergency room visits (1.19), outpatient visits (1.18), and skilled nursing facility stay (1.35). DISCUSSION/SIGNIFICANCE OF IMPACT: Patients with idiopathic pulmonary fibrosis experience increased co-morbidity and healthcare resource utilization compared to controls. This increased burden extends beyond the index-period as previously documented and is true for a large real-world cohort. CONFLICT OF INTEREST DESCRIPTION: NA

CD4 count is a prognostic marker in persons living with HIV and non-small cell lung cancer in the Bronx

Madelyn Klugman ${ }^{1}$, Melissa Fazzari, Mindy Ginsberg, Thomas

Rohan, David Hanna, Jonathan Shuter, and H. Dean Hosgood, III ${ }^{1}$ Albert Einstein College of Medicine

OBJECTIVES/GOALS: There is a high burden of lung cancer in persons living with HIV (PLWH). The role that HIV status, by levels of immune function and viral load, has on survival from lung cancer is not fully understood. The study's objectives were to assess 1) the association of HIV with survival in non-small cell lung cancer (NSCLC) and 2) prognostic factors in PLWH with NSCLC. METHODS/STUDY POPULATION: Participants were from a cohort of lung cancer patients diagnosed between 2004-2017 in the Bronx, NY, with vital status ascertainment at least annually. We compared survival from NSCLC diagnosis between HIV-negative patients (HIV-, $\mathrm{N}=2881$ ) and PLWH $(\mathrm{N}=88)$, using Cox regression, accounting for clinical and sociodemographic factors including smoking status. In three separate comparisons to HIV-, PLWH were dichotomized by CD4 count $(<200$ vs. $\geq 200$ cells $/ \mu \mathrm{L}$ ), CD4/CD8 ratio (median, $<0.43$ vs. $\geq 0.43$ ) and HIV viral load (VL) suppression ( $<75$ vs. $\geq 75$ copies $/ \mathrm{mL}$ ). In PLWH only, we assessed the relationships of CD4 count, CD4/CD8 ratio, and VL at diagnosis with survival adjusting for age, sex, and cancer stage. CD4 count and CD4/CD8 ratio were also examined as timevarying variables using a counting process approach. RESULTS/ ANTICIPATED RESULTS: PLWH were younger (median 56 years, IQR 51-52 vs. 68, IQR 60-76) and more likely to be current smokers (58\% vs. $37 \%$ ) at diagnosis than HIV-patients. Median survival was lower in PLWH [1.1 years, 95\% confidence interval (95\%CI): 0.6-1.3] than in HIV- [1.6 (1.5-1.7)]. Survival comparing PLWH with higher CD4/CD8 to HIV- was similar [hazard ratio (HR), 95\%CI: 0.63 (0.37-1.07)], but those with lower CD4/CD8 experienced worse survival $(\mathrm{HR}=1.74,95 \% \mathrm{CI}$ : 1.07-3.89). Among PLWH, having a CD4 count $<200$ cells $/ \mu \mathrm{L}$ was associated with over twice the risk of death compared to those with CD $4 \geq 200$ cells $/ \mu \mathrm{L}$ ( $\mathrm{HR}=2.37,95 \% \mathrm{CI}: 1.14$ 4.92). VL and CD4/CD8 ratio were not associated with survival. Lower time-updated CD4 count was also associated with worse survival $(\mathrm{HR}=2.19$ for $\mathrm{CD} 4<200$ vs. $>200$ cells $/ \mu \mathrm{L}, 95 \% \mathrm{CI}$ : $1.16-4.13)$. DISCUSSION/SIGNIFICANCE OF IMPACT: Among persons with NSCLC, CD4/CD8 ratio nearest diagnosis was shown to distinguish mortality risk in PLWH compared with HIV- patients. In addition, PLWH with low CD4 had worse prognosis than PLWH who had higher CD4 counts. These results suggest HIV immune status to be an essential component influencing survival in lung cancer.

4223

\section{Changes in positive predictive value of cervical cytology following HPV vaccination}

Deanna Teoh, Teoh, University of Minnesota ${ }^{1}$, Gwiwon Nam², Shalini Kulasingam, $\mathrm{PhD}^{2}$, and Rachel I. Vogel, $\mathrm{PhD}^{2}$

${ }^{1}$ University of Minnesota CTSI; ${ }^{2}$ University of Minnesota

OBJECTIVES/GOALS: To determine if current U.S. HPV vaccination rates have decreased dysplasia prevalence enough to decrease the positive predictive value (PPV) of abnormal cervical cytology. METHODS/STUDY POPULATION: This retrospective cohort study comprised a chart review of all patients 21-35 years of age who had at least 1 Pap test result within MHealth/Fairview 20162018. HPV vaccination data, cervical cancer screening data and dysplasia results were abstracted. Vaccinated was defined as receiving at least 1 dose of HPV vaccine, with subgroup analyses performed for those completing vaccination per ACIP guidelines and by age of initiation dichotomized as $21+$ years versus $<21$ years. RESULTS/ ANTICIPATED RESULTS: 49,764 patients meeting study criteria were identified. Among the entire study population, $10 \%$ had abnormal cytology results during the study period. Among the 4,928 patients with abnormal cytology, PPV for CIN2+ was lower among vaccinated individuals $(13 \%$ vs. $18 \%$; $<<0.0001)$. Among vaccinated 